\title{
The usefulness of intraoperative consultation for the diagnosis of borderline ovarian tumors
}

\author{
Rosa A. Salcedo-Hernández ${ }^{1}$, David F. Cantú-de-León ${ }^{2}$, Delia Pérez-Montiel ${ }^{3}$, Leticia García-Pérez ${ }^{1}$, \\ Leonardo S. Lino-Silva ${ }^{3}$, César Zepeda-Najar ${ }^{4}$, Salim A. Barquet-Muñoz ${ }^{1}$ \\ ${ }^{1}$ Surgical Oncology, National Cancer Institute, Mexico City, Mexico; ${ }^{2}$ Gynecologic Oncology, National Cancer Institute, Mexico City, Mexico; \\ ${ }^{3}$ Surgical pathology, National Cancer Institute, Mexico City, Mexico; ${ }^{4}$ Surgical oncology, Hospital ángeles Tijuana, Tijuana, Baja California Norte, \\ Mexico \\ Contributions: (I) Conception and design: All authors; (II) Administrative support: C Zepeda-Najar, DF Cantú-de-León; (III) Provision of study \\ materials or patients: All authors; (IV) Collection and assembly of data: RA Salcedo-Hernández; (V) Data analysis and interpretation: RA Salcedo- \\ Hernández, LS Lino-Silva; (VI) Manuscript writing: All authors; (VII) Final approval of manuscript: All authors. \\ Correspondence to: Rosa A. Salcedo-Hernández, MSc. Gynecological Cancers Division, Instituto Nacional de Cancerología de México (Mexico’s \\ National Cancer Institute), Av. San Fernando \# 22, Sección XVI, Tlalpan, Mexico City, Mexico. Email: gyneonco.incan@gmail.com.
}

Background: Borderline ovarian tumors (BTs) must be recognized during the surgery by intraoperative consultation (IOC) to guide surgical treatment; however, this diagnosis can be imprecise. Therefore, this study aimed to evaluate the diagnostic accuracy of IOC for the diagnosis of BT.

Methods: A retrospective cohort study was carried out including all women diagnosed with a pelvic tumor consecutively surgically treated from 2005 to 2015 with IOC. We calculated the sensitivity, specificity, positive predictive value (PPV), negative predictive value (NPV), and likelihood ratios (LR) for the IOC and BTs.

Results: A total of 758 patients were enrolled, the median age was 44 years, the median tumor size was $11.8 \mathrm{~cm}$, and the median CA-125 levels were $45.65 \mathrm{U} / \mu \mathrm{L}$. After IOC, 458 (64.1\%) cases were diagnosed as benign, $111(14.7 \%)$ as BT, and $161(21.2 \%)$ as malignant. The definitive diagnosis was a benign tumor in 448 (59.1\%) cases, BT in 110 (14.5\%), and 200 (26.4\%) cases were malignant. The diagnostic accuracy of the IOC for BT diagnosis was $89.8 \%$ (sensitivity $=65.5 \%$, specificity $=93.9 \%$ ). The diagnosis performance of IOC for the diagnosis between BT and benign tumors $(n=546)$ had a sensitivity of $69.9 \%$, a specificity of $98.4 \%$, and a diagnostic accuracy of $84 \%$; meanwhile for the diagnosis between BT and malignant tumors $(\mathrm{n}=242$ ) IOC had a sensitivity of $92.3 \%$, a specificity of $81.7 \%$, and a diagnostic accuracy of $87 \%$.

Conclusions: For practitioners, knowing the accuracy and limitations of the IOC for BT enables the better selection of cases to perform a complete staging surgery.

Keywords: Ovarian cancer; borderline ovarian tumors (BTs); intraoperative consultation (IOC); pathology; diagnostic accuracy

Submitted May 14, 2020. Accepted for publication Nov 02, 2020.

doi: 10.21037/atm-20-3932

View this article at: http://dx.doi.org/10.21037/atm-20-3932

\section{Introduction}

Borderline ovarian tumors (BTs) represent between $10 \%$ to $20 \%$ of epithelial neoplasms of the ovary and are characterized by complex structures with mild cytological atypia and the absence of stromal infiltration (1). When
BT occurs in young women, they have a good prognosis and the treatment is a surgery similar to that described for ovarian carcinoma; but fertility-sparing surgery can be offered without affecting oncologic outcomes (2). The diagnosis of this neoplasm is impossible prior to surgery, so it is necessary to diagnose it during surgery using an 
intraoperative consultation (IOC).

The IOC is a pathologic evaluation of the ovarian tumor during surgery; it consists of observing any combination of the macroscopic features of the specimen, cytological examination, and interpretation of frozen sections. In a Cochrane systematic review, the authors compared the IOC and final reports and found that when the IOC diagnosis is a BT, it is less accurate (inadequate sampling and interpretation error); for example, $21 \%$ of patients with IOC diagnoses of BT had a diagnosis of cancer in the final report (3).

Several studies have evaluated the accuracy of the IOC in BT diagnosis with varying results, with the sensitivity ranging between values of $30 \%$ to $61 \%$, a specificity of $90 \%$ to $94 \%$, and diagnostic accuracies between $69.5 \%$ and $76.8 \%(4-6)$.

Errors in the IOC diagnosis are typically due to the type of epithelium, inadequate sampling, technical problems in the preparation of slides, inappropriate interpretation, and the experience of the pathologist (7).

Because it is impossible to diagnose BT preoperatively using radiological imaging and/or tumor markers, the surgeon depends on real-time integration of these data, the age of the patient, and a reliable pathologist to reach a diagnosis during the IOC and determine the necessary extent of the surgery, so as not to over- or under-treat patients with pelvic tumors. For example, a false-negative diagnosis could cause that the patient will not be adequately staged, so the patient will probably require repeat surgery; on the contrary, a false positive in a young patient will cause a series of unnecessary surgical procedures that will compromise her reproductive and hormonal function.

In this study, we evaluated the diagnostic accuracy of the IOC in the diagnosis of BT at a high-volume center. We present the following article in accordance with the STROBE reporting checklist (available at http://dx.doi. org/10.21037/atm-20-3932).

\section{Methods}

A retrospective cohort study was carried out. All women $>18$ years with a diagnosis of pelvic tumor treated with surgery with IOC in ovarian tumors in our hospital consecutively from 2005 to 2015 , were selected.

Patients for whom the following data were complete and available were included in the present study: clinical information, IOC report, final histopathological report, pre-surgery tumor marker profile, and preoperative images.
The specific variables collected were age, radiological features of the tumor (size, presence of septa, the complexity of the lesion, and the presence of free fluid in the cavity), and CA125 level. Cases were categorized as benign, BT, or carcinoma.

\section{Statistical analysis}

Descriptive statistics were used to summarize the general characteristics of the sample. We used the KolmogorovSmirnov test on the numerical variables to test for normal distribution. Median and interquartile ranges were used to represent numerical variables. For nominal variables, we used values and percentages. Sensitivity (Se), specificity $(\mathrm{Sp})$, positive predictive value (PPV), negative predictive value (NPV), and likelihood ratios (LR) were calculated to assess the diagnostic accuracy of IOC. We performed the calculations using contingency tables and applying the following definitions: (I) Se is defined as the probability that a test is positive when the patient has the disease, calculated using the formula: $\mathrm{Se}=$ (true positives) $/($ true positives + false negatives); (II) $\mathrm{Sp}$ is defined as the probability of having a negative result when the patient does not have the disease, calculated with the formula: $S p=$ (true negatives) $/$ (true negatives + false negatives); (III) PPV is the probability of having the disease when the test is positive, calculated with the formula: PPV $=$ (true positives)/(true positives + false positives); (IV) NPV is the probability of not having the disease when the test is negative, calculated with the formula: NPV $=$ (true negatives)/(true negatives + false negatives); (V) LR is the ratio between the possibility of observing a result in patients with the disease in question versus the possibility of this result in patients without the disease, calculated by the formula: LR $(+)=(\mathrm{Se}) /(1-\mathrm{Sp})$, and the LR $(-)=(1-\mathrm{Se}) /(\mathrm{Sp})$. The LR is particularly helpful when making clinical decisions based on a diagnostic test because it is inherent to the test and independent of disease prevalence.

Moreover, the proportion of discordant studies that led to the modification of the medical decision (over- and under-treatment) was measured. For statistical analysis, we used STATA software version 14 (StataCorp LLC., College Station, TX, USA). For all tests, a $\mathrm{P}$ value $<0.05$ was considered reflective of statistical significance.

The study was conducted in accordance with the Declaration of Helsinki (as revised in 2013) and was approved by our institutional ethics and research committee (Register Rev/0058/19). Being a retroSpective study, our 
Table 1 General features of 758 patients with ovarian lesions who underwent surgery + IOC [2005-2015, National Cancer Institute (Mexico)]

\begin{tabular}{lc}
\hline Characteristic & Values \\
\hline Age (years), median (interquartile range) & $44(35.0-53.0)$ \\
Size by radiology (cm), median & $11.8(7.5-17.0)$ \\
(interquartile range) & \\
Radiology features, $\mathrm{n}(\%)$ & $122(16.18)$ \\
Simple cyst & $601(79.71)$ \\
Complex cyst & $31(4.11)$ \\
Solid tumor & $301(39.32)$ \\
Septa & $130(17.24)$ \\
Ascitis, $\mathrm{n}$ (\%) & $45.65(15.45-140.5)$ \\
Seric level of CA125 (U/ $\mathrm{LL})$, median \\
(interquartile range) \\
IOC diagnosis, $\mathrm{n}$ (\%) \\
Benign & $184(24.3)$ \\
Borderline tumor & $16(2.11)$ \\
Malignant tumor & $486(64.1)$ \\
Metastatic neoplasm & $111(14.7)$ \\
Final histopathology report, $\mathrm{n}(\%)$ & $148(19.5)$ \\
Benign & $13(1.7)$ \\
Borderline tumor & \\
Malignant tumor & $448(59.1)$ \\
Metastatic neoplasm & $110.5)$ \\
\hline
\end{tabular}

Table 2 Case comparison between frozen section diagnosis and final diagnosis

\begin{tabular}{lccc}
\hline & \multicolumn{3}{c}{ Final diagnosis } \\
\cline { 2 - 4 } IOC diagnosis & $\begin{array}{c}\text { Benign } \\
(\mathrm{n}=448)\end{array}$ & $\begin{array}{c}\text { Borderline } \\
(\mathrm{n}=110)\end{array}$ & $\begin{array}{c}\text { Malignant } \\
(\mathrm{n}=200)\end{array}$ \\
\hline Benign $(\mathrm{n}=486)$ & 445 & 6 & 35 \\
Borderline $(\mathrm{n}=111)$ & 1 & 72 & 38 \\
Malignant $(\mathrm{n}=161)$ & 2 & 32 & 127 \\
\hline
\end{tabular}

research did not impoSe any risk for the patients and their management, and preServes their confidentiality; it was thus not necessary to obtain an informed conSent letter.

\section{Results}

We reviewed 758 records of patients operated at our institution with a diagnosis of a pelvic tumor from 2005 to 2015. No cases with IOC were deferred. Tables 1,2 summarize the general characteristics of the patients. Despite the same percentage of BT being diagnosed in the IOC as in the final report, these were not the same cases, given the concordance rate of $64.8 \%(72 / 111)$. Of the discordant cases diagnosed as a BT in the IOC, $27 \%$ (30/111) were carcinomas, $6.3 \%$ were benign, and $1.8 \%$ were metastatic in the final histopathological report. With $\mathrm{BT}$ as the expected diagnosis, there was a $5.1 \%$ falsenegative rate, meaning that they were misdiagnosed in the IOC (benign or carcinoma) and were reported as a BT in the final report.

Sensitivity and specificity analyses included not only evaluating the IOC for the diagnosis of BT but also analyzing its diagnostic utility compared with the diagnosis of a benign or malignant neoplasm (Table 3). The diagnostic accuracy of the IOC for BT was $89.8 \%$.

Analyzing the clinical characteristics of BT versus benign tumors and carcinomas revealed differences in tumor size and marker level (CA-125) between BT and the benign tumors (Tables 4,5). The diagnoses of false positives in the IOC were analyzed, and we found that metastatic mucinous adenocarcinoma and mucinous adenocarcinomas of the ovary were the main sources of this discordance (Table 6).

Misdiagnosis led to inadequate treatment for $1.1 \%$ of the patients. False-positive cases were those had a BT report in the IOC and underwent staging surgery, with the definitive diagnosis of benign tumors. There was sub-treatment or incomplete treatment in $5.1 \%$ of cases. In $27 \%$ of the patients with a false-positive diagnosis, the final report was carcinoma. Then, patients were adequately treated since the international guidelines recommended that patients with BT undergo the same staging surgery as patients with carcinoma.

\section{Discussion}

In our series, $14.6 \%$ of pelvic tumors were diagnosed as a BT in the final histopathological report. The IOC diagnosis had high precision, close to $90 \%$; however, $27 \%$ of the tumors diagnosed as BT during the IOC were carcinomas in the final report, which is much higher than 
Table 3 Diagnostic utility of IOC

\begin{tabular}{lccc}
\hline Statistical measure & $\begin{array}{c}\text { Overall diagnosis of ovarian } \\
\text { tumors }(\mathrm{n}=758)\end{array}$ & $\begin{array}{c}\text { Diagnosis between borderline and } \\
\text { benign tumors }(\mathrm{n}=546)\end{array}$ & $\begin{array}{c}\text { Diagnosis between borderline and } \\
\text { malignant tumors }(\mathrm{n}=242)\end{array}$ \\
\hline Sensibility & 65.45 & 69.90 & 92.31 \\
Specificity & 93.98 & 98.42 & 81.71 \\
LR+ & 10.88 & 44.23 & 5.05 \\
LR- & 0.37 & 0.31 & 0.09 \\
AUC $(95 \% \mathrm{Cl})$ & $0.79(0.77-0.82)$ & $0.84(0.79-0.89)$ & $0.87(0.83-0.91)$ \\
\hline
\end{tabular}

LR, likelihood ratio; AUC, area under the curve.

Table 4 Comparison between radiological findings and tumor markers between ovarian borderline tumors and ovarian benign tumors

\begin{tabular}{|c|c|c|c|}
\hline Characteristic & Benign $(n=448)$ & Borderline $(n=108)$ & $\mathrm{P}$ \\
\hline Size by radiology $(\mathrm{cm})$, median (interquartile range) & $9.5(6.5-14)$ & $16(11.0-24.0)$ & $<0.01$ \\
\hline Radiology features, n (\%) & & & 0.03 \\
\hline Simple cyst & $80(17.86)$ & $28(25.93)$ & \\
\hline Solid tumor & $11(2.46)$ & $2(1.85)$ & \\
\hline Septa, n (\%) & $176(39.29)$ & $51(47.22)$ & 0.13 \\
\hline Ascitis, $\mathrm{n}(\%)$ & $48(10.71)$ & $28(25.93)$ & $<0.01$ \\
\hline Seric level of CA125, median (Interquartile range) & $22.1(9.7-71.9)$ & $106(41.7-353)$ & $<0.001$ \\
\hline
\end{tabular}

Table 5 Comparison between radiological findings and tumor markers between ovarian borderline tumors and ovarian malignant tumors

\begin{tabular}{|c|c|c|c|}
\hline Characteristic & Malignant $(n=182)$ & Borderline $(n=108)$ & $\mathrm{P}$ \\
\hline Size by radiology $(\mathrm{cm})$, median (interquartile range) & $15(11.0-19.0)$ & $16(11.0-24.0)$ & 0.02 \\
\hline Radiology features, n (\%) & & & $<0.01$ \\
\hline Simple cyst & $12(6.59)$ & $28(25.93)$ & \\
\hline Solid tumor & $16(8.79)$ & $2(1.85)$ & \\
\hline Septa, n (\%) & $68(37.36)$ & $51(47.22)$ & 0.10 \\
\hline Ascitis, $\mathrm{n}(\%)$ & $48(26.37)$ & $28(25.93)$ & 0.93 \\
\hline Seric level of CA125, median (interquartile range) & $107(49.4-272.5)$ & $106(41.7-353)$ & 0.86 \\
\hline
\end{tabular}

that reported in a Cochrane review (21\%). These results are likely associated with the medical team because a large proportion of the IOC cases are evaluated by pathologists in training and pathologists specializing in non-gynecological areas who have less experience with this type of neoplasms $(3,8)$. In our statistical analysis, we found that the IOC is useful for the diagnosis of BT (LR+ 10.8); however, its utility increased when used to differentiate a BT from a 
Table 6 Final diagnosis of discordant cases $(n=39)$ diagnosed during the IOC as BTs by the pathologist

\begin{tabular}{lcc}
\hline Final diagnosis & Number of cases (\%) & Cause of discrepance \\
\hline Mucinous carcinoma & $8(20.51)$ & Sampling \\
Low-grade serous carcinoma & $7(17.95)$ & Sampling \\
Endometrioid carcinoma & $7(17.95)$ & Frozen section artifact \\
Clear cell carcinoma & $4(10.25)$ & Sampling \\
Serous cystadenofibroma (extensive necrosis) & $4(10.25)$ & Sampling \\
Metastasis, appendix mucinous neoplasm & $2(5.13)$ & Abscence of clinical information \\
High-grade serous carcinoma & $1(2.56)$ & Sampling \\
Low-grade seromucinous carcinoma & $1(2.56)$ & Sampling \\
Mixed endometrioid/clear cell carcinoma & $1(2.56)$ & Sampling \\
Intraepithelial mucinous carcinoma & $1(2.56)$ & Sampling \\
Metastasis, gastric signet ring cell carcinoma & $1(2.56)$ & $1(2.56)$ \\
Endometriosic cyst & $1(2.56)$ & Abscence of clinical information \\
Mucinous cysteadenofibroma & & Interpretation
\end{tabular}

benign one $(L R+44.23)$. In contrast, its value decreased when diagnosing a BT by ruling out carcinoma (LR+ 5.05), because the morphological characteristics of $\mathrm{BT}$ and carcinomas are identical, and ruling out stromal invasion hinges on the sampling experience of the pathologist (1).

In our series, we observed that the most pronounced discrepancies between the IOC and the final report were in mucinous tumors (benign, carcinomas, or metastasic), which is consistent with the literature. There are reports that ovarian mucinous neoplasms decrease the sensitivity and specificity of the IOC due to tumor heterogeneity $(9,10)$ However, there was also considerable disagreement in the diagnosis of low-grade serous tumors, because up to $20 \%$ of serous BT may contain foci or areas that obviously cannot be evaluated during the IOC due to size and limited sampling (11). The discrepancy between the diagnosis of serous tumors (i.e., serous BT and serous carcinoma) and endometrioid carcinoma (in particularly high-grade ones) can be explained because sometimes serous neoplasms display a glandular pattern and endometrioid carcinomas can show a papillary pattern, pseudopapillary growth, solid nests, and marked pleomorphism that require additional studies (e.g., immunohistochemical) to establish the difference (12).

Overtreatment and inadequate treatment were observed in $1.1 \%$ of the cases. Very few patients had a BT identified in the IOC that the later staging surgery revealed to be a benign or metastatic tumor for the final report; this percentage was tiny and, as such, we consider it acceptable. The sub-treatment of patients with false negatives accounted for $5.1 \%$ of all our cases, mainly because the IOC diagnosis was a benign tumor in $79.4 \%$ of cases or a metastatic tumor found upon definitive diagnosis to be a BT. The $27 \%$ for whom the final report included a carcinoma diagnosis were adequately treated since the international guidelines recommended that patients with $\mathrm{BT}$ undergo the same staging surgery as patients with carcinoma; however, this is unlikely to be a fertility-sparing surgery (13).

Diagnosing BT is crucial because this determines the immediate treatment course of patients and enables avoiding Sub- or overtreatment, and can offer fertilitypreserving procedures to candidate patients without affecting their survival. Our population had few cases of fertility-sparing surgery. Potential explanations of this are that our patients are typically not young, the median age of the first pregnancy in our country is adolescent age and, before 2010, this option was very rarely offered in our service.

To date, findings from ultrasound or tomography (e.g., the presence of septa, vascularity, resistance, pulsatility index, and velocimetry) have been described, but not yet proven useful (14). Prediction models combining tumor markers, patient age, and ultrasonographic findings have been developed, but continue to fall short of success (15). 
In the present study, we found that tumor size (measured in the imaging study) varied between BTs and the benign tumors and carcinomas. BTs were more massive than carcinomas because of their slow and indolent growth. The carcinomas presented more frequently in the imaging analysis as complex or solid adnexal lesions. On the other hand, there was no difference in the age of presentation between BTs, carcinomas, and benign tumors, despite the fact that the median age of diagnosis for ovarian cancer is 61 years old (13). In the present series, the median age of patients with carcinomas was 49 years compared with BT's median of 44 years. Our findings suggest that age and ultrasound findings are of little value in the diagnosis of this pathology.

This study has the limitations of being retrospective; however, the sample size was large, with complete data that allowed us to perform a comprehensive analysis. Another strength is that a second pathology review was included when necessary and obtained accurate results. This is the first study to extend this type of analysis to a specific population of Mexico. Working in a National Reference Center allow us to make recommendations for sample handling, and proposing training programs to pathologists specializing in pelvic neoplasms.

\section{Conclusions}

The IOC represents a challenge for any pathologist, and pelvic lesions are no exception. For the practitioners, knowing the accuracy and limitations of the IOC for BT will allow them to best select cases after talking to the patients to make a well-informed joint decision and consider those who will eventually provide a conservative or more radical treatment.

\section{Acknowledgments}

Funding: None.

\section{Footnote}

Reporting Checklist: The authors have completed the STROBE reporting checklist. Available at http://dx.doi. org/10.21037/atm-20-3932

Data Sharing Statement: Available at http://dx.doi. org/10.21037/atm-20-3932
Peer Review File: Available at http://dx.doi.org/10.21037/ atm-20-3932

Conflicts of Interest: All authors have completed the ICMJE uniform disclosure form (available at http://dx.doi. org/10.21037/atm-20-3932). The authors have no conflicts of interest to declare.

Ethical Statement: The authors are accountable for all aSpects of the work in ensuring that questions related to the accuracy or integrity of any part of the work are appropriately investigated and resolved. The study was conducted in accordance with the Declaration of Helsinki (as revised in 2013) and was approved by our institutional ethics and research committee (Register Rev/0058/19). Being a retroSpective study, our research did not impoSe any risk for the patients and their management, and preServes their confidentiality; it was thus not necessary to obtain an informed conSent letter.

Open Access Statement: This is an Open Access article distributed in accordance with the Creative Commons Attribution-NonCommercial-NoDerivs 4.0 International License (CC BY-NC-ND 4.0), which permits the noncommercial replication and distribution of the article with the strict proviso that no changes or edits are made and the original work is properly cited (including links to both the formal publication through the relevant DOI and the license). See: https://creativecommons.org/licenses/by-nc-nd/4.0/.

\section{References}

1. Ouldamer L, Lacoste C, Cormier B, et al. Is there a justification for hysterectomy in patients with borderline ovarian tumors? Surg Oncol 2016;25:1-5.

2. Huang $Y$, Zhang W, Wang Y. The feasibility of fertilitysparing surgery in treating advanced-stage borderline ovarian tumors: A meta-analysis. Taiwan J Obstet Gynecol 2016;55:319-25.

3. Ratnavelu ND, Brown AP, Mallett S, et al. Intraoperative frozen section analysis for the diagnosis of early stage ovarian cancer in suspicious pelvic masses. Cochrane Database Syst Rev 2016;3:CD010360.

4. Pinto PB, Andrade LA, Derchain SF. Accuracy of intraoperative frozen section diagnosis of ovarian tumors. Gynecol Oncol 2001;81:230-2.

5. Boriboonhirunsarn D, Sermboon A. Accuracy of frozen 
section in the diagnosis of malignant ovarian tumor. J

Obstet Gynaecol Res 2004;30:394-9.

6. Gultekin E, Gultekin OE, Cingillioglu B, et al. The value of frozen section evaluation in the management of borderline ovarian tumors. J Cancer Res Ther 2011;7:416-20.

7. Stewart CJ, Brennan BA, Hammond IG, et al. Intraoperative assessment of ovarian tumors: a 5-year review with assessment of discrepant diagnostic cases. Int J Gynecol Pathol 2006;25:216-22.

8. Basaran D, Salman MC, Calis P, et al. Diagnostic accuracy of intraoperative consultation (frozen section) in borderline ovarian tumours and factors associated with misdiagnosis. J Obstet Gynaecol 2014;34:429-34.

9. Bige O, Demir A, Saygili U, et al. Frozen section diagnoses of 578 ovarian tumors made by pathologists with and without expertise on gynecologic pathology. Gynecol Oncol 2011;123:43-6.

10. Basaran D, Salman MC, Boyraz G, et al. Accuracy of intraoperative frozen section in the evaluation of patients

Cite this article as: Salcedo-Hernández RA, Cantú-de-León DF, Pérez-Montiel D, García-Pérez L, Lino-Silva LS, Zepeda-Najar C, Barquet-Muñoz SA. The usefulness of intraoperative consultation for the diagnosis of borderline ovarian tumors. Ann Transl Med 2021;9(3):261. doi: 10.21037/atm-20-3932 with adnexal mass: retrospective analysis of 748 cases with multivariate regression analysis. Pathol Oncol Res 2015;21:113-8.

11. Sukumaran R, Somanathan T, Mathews A, et al. Role of frozen section in intraoperative assessment of ovarian masses: a tertiary oncology center experience. Indian J Surg Oncol 2014;5:99-103.

12. Garg K, Soslow RA. Strategies for distinguishing low-grade endometrioid and serous carcinomas of endometrium. Adv Anat Pathol 2012;19:1-10.

13. National Comprehensive Cancer Network. Ovarian Cancer (Version 1.2019). Available online: https://www. nccn.org/professionals/physician_gls/pdf/ovarian.pdf

14. Pascual MA, Tresserra F, Grases PJ, et al. Borderline cystic tumors of the ovary: gray-scale and color Doppler sonographic findings. J Clin Ultrasound 2002;30:76-82.

15. Chen J, Chang C, Huang HC, et al. Differentiating between borderline and invasive malignancies in ovarian tumors using a multivariate logistic regression model. Taiwan J Obstet Gynecol 2015;54:398-402. 